\title{
Translating the Diabetes Control and Complications Trial (DCCT) into clinical practice: overcoming the barriers
}

\section{B. Zinman}

Samuel Lunenfeld Research Institute, Mount Sinai Hospital, Department of Medicine, University of Toronto, Toronto, Canada

The Diabetes Control and Complications Trial (DCCT) [1] and other controlled randomized prospective studies [2-4] have demonstrated conclusively that intensive diabetes management, which results in improvement in haemoglobin $\mathrm{A}_{1 \mathrm{c}}$, dramatically reduces the rate of development and the progression of diabetic retinopathy, nephropathy and neuropathy. The DCCT, the largest of these studies was the only one also to address the issue of primary prevention of long-term complications. The DCCT determined, in 1441 insulin-dependent diabetic (IDDM) patients, that intensive therapy would result in a risk reduction for the development of retinopathy of $76 \%$ in the primary prevention group; a risk reduction of $54 \%$ for progression of retinopathy in the secondary intervention group; a risk reduction for development of microalbuminuria of $39 \%$ and clinical grade proteinuria of $50 \%$. There was also a $60 \%$ reduction in the risk of developing clinical neuropathy. Unfortunately, these striking benefits were also associated with two major undesirable adverse occurrences, namely, an increased risk of severe hypoglycaemia (two- to threefold) and excessive weight gain. Nonetheless, on balance, it is clear that intensive therapy is associated with dramatic benefits in relationship to the management of IDDM and, as a consequence, should be considered as the treatment of choice for most IDDM patients.

The extrapolation of observations from studies performed on highly motivated patients in the setting of an academic health care delivery programme to everyday clinical practice must be done cautiously. Indeed, it is not always certain that the treatment

Corresponding author: Dr. B. Zinman, Mount Sinai Hospital, 600 University Avenue, Suite 782, Toronto, Ontario M5G 1X5, Canada

A bbreviations: IDDM, Insulin-dependent diabetes mellitus; DCCT, Diabetes Control and Complications Trial. goals achieved in a research setting can be safely translated to clinical practice. The implementation of intensive therapy in a more heterogeneous population may be associated with increased risk and, perhaps as yet undefined, long-term adverse effects. In this article, the barriers associated with implementing intensive insulin therapy, similar to those employed in the DCCT [5], in a clinical setting, will be examined.

Implementing intensive diabetes management in IDDM

The barriers associated with implementing intensive therapy are listed in Table 1. Each of these will be reviewed.

\section{The therapeutic regimen}

Unfortunately, with our current therapeutic regimens, physiologic insulin replacement remains an elusive goal [6] and would require pulsatile portal insulin release responsive to feedback control and modulated by various hormones. We are limited to the subcutaneous routes of insulin adminstration for the vast majority of patients. The development of basal bolus therapy with multiple daily injections or continuous subcutaneous insulin infusion, although superior to conventional therapy consisting of intermediate and regular insulin combinations often given once or twice daily, does not duplicate physiologic requirements. Nonetheless, when intensive insulin treatment regimens are coupled with frequent capillary blood glucose monitoring and a quantitative method for estimating carbohydrate intake, remarkable glycaemic control can be achieved. It must be appreciated that many variables will determine the 
Table 1. Barriers to implementing intensive therapy

- Therapeutic regimen

- Health care team

- Financial consideration

- The Patient

Table 2. Intensive diabetes management

- is not user friendly

- is dependent on frequent capillary blood glucose monitoring

- requires a multiple daily injection regimen or insulin pump therapy

- requires adherence to a dietary adjustment strategy (e.g. carbohydrate counting)

Table 3. The health care team

\begin{tabular}{ll}
\hline Members: & Nurse educator \\
& Dietitian \\
& Patient \\
& Physician \\
Philosophy: & Open and ongoing communication \\
& Joint decision-making \\
& Unified message \\
& The patient is the problem solver \\
\hline
\end{tabular}

Table 4. DCCT annual cost of diabetes management (US dollars)

\begin{tabular}{ll}
\hline Conventional therapy & 1666 \\
Intensive therapy $^{\mathrm{a}}$ & 2848
\end{tabular}

a Weighted average of the costs of multiple daily injections and continuous subcutaneous insulin infusion

absorption characteristics of a particular dose of insulin, including the injection site, depth of the injection, the ambient temperature and the performance of exercise, to list some of the obvious factors. It is thus not surprising that frequent adjustment of the therapeutic regimen on a day-to-day and dose-by-dose basis must be mastered to achieve an optimal result. Our current methods of replacing insulin in IDDM are not user-friendly and require a comprehensive educational approach to allow the patients to achieve effective results (Table 2).

\section{The health care team}

The safe and effective implementation of intensive therapy with the aim of achieving normoglycaemia requires a health care team consisting of a nurse educator, a dietitian and a physician, all working with the patient. The goal of this team is to provide the patient with the education and skills necessary for the successful implementation of intensive diabetes management. For the health care team to work effectively, the group must agree to work in a collaborative manner. This can best be done if they adhere to a common philosophy as outlined in Table 3.

\section{Financial considerations}

A detailed analysis of the lifetime benefits and costs of intensive therapy, as practiced by the DCCT has recently been published [8]. The main costs related to intensive therapy are the result of increased capillary blood glucose monitoring and more frequent interactions with the health care team, and adverse effects of therapy (hypoglycaemia and weight gain) (Table 4). It is evident that although health care costs are initially higher with intensive therapy, with a longer duration of diabetes and the increased prevalence of long-term complications (particularly renal disease), the cost:benefit analysis of intensive therapy becomes significantly more favourable. In the context of health care delivery for people with diabetes, it is important to have a long-term view. It is the obligation of both patients and health care providers to convince government and other health care agencies of the benefits of intensive therapy on improving health outcome and quality of life. In terms of quality adjusted life years (QALY), intensive therapy costs 19,987 US dollars per QALY gained [8]. This compares very favourably with commonly accepted therapeutic modalities, such as the treatment of hypertension, and by most standards is a strong endorsement to implement intensive therapy.

\section{The patient}

Intensive diabetes management will only be successful when the patient (the client) is well-educated, motivated to pursue the goals of intensive therapy and is an integrated member of the health care team. It is important to have a relationship that allows the patient to be honest with his/her health care providers and become skilled in modifying the treatment regimen in an effective manner so that the individual may continue to pursue a satisfactory lifestyle. At the outset it is important to establish the goals of therapy. The factors that will determine these goals will include, in addition to the patient's motivation, the general health of the individual, the status of their complications, their age and expected longevity, the availability of family support and the risk of severe hypoglycaemia. Patients who have psychiatric disturbances and/or eating disorders are often extremely difficult to manage in an intensive manner. Ultimately, if intensive therapy is implemented successfully and metabolic outcomes are similar to those achieved in the DCCT, significant life-long improvement in 
Table 5. Intensive therapy results in additional complicationfree years

\begin{tabular}{lr}
\hline Proliferative retinopathy & 14.7 \\
Blindness & 7.7 \\
Albuminuria & 9.7 \\
End-stage renal disease & 5.8 \\
Neuropathy & 10.9 \\
Lower extremity amputation & 5.6 \\
First significant complication & 15.3 \\
Life & 5.1 \\
\hline
\end{tabular}

outcome can be expected. In Table 5 this improvement in outcome is expressed as additional complication-free years.

\section{Conclusions}

The translation of research findings to the clinical setting is an important and natural consequence of clinical investigation. In this article we have attempted to highlight some of the barriers that have to be addressed and considered when implementing intensive therapy in a clinical setting. We have not dealt with the question of extrapolating results of glycaemic control on long-term complications in IDDM to individuals with non-insulin-dependent diabetes. This is a different issue and the United Kingdom Prospective Diabetes Study will provide information addressing the benefits and risks of improved glycaemic control in newly diagnosed individuals with this disease [7].

\section{References}

1. The Diabetes Control and Complications Trial Research Group (1993) The effect of intensive treatment of diabetes on the development and progression of long-term complications in insulin dependent diabetes mellitus. N Engl J Med 329: 977-986

2. Lauritzen T, Frost-Larsen K, Larsen H-W, Deckert T (1985) Two-year experience with continuous subcutaneous insulin infusion in relation to retinopathy and neuropathy. Diabetes 34[Suppl]:74-79

3. Reichard P, Nilsson B-Y, Rosenqvist U (1993) The effect of long-term intensified insulin treatment on the development of microvascular complications of diabetes mellitus. N Engl J Med 329: 304-309

4. Brinchmann-Hansen O, Dahl-Jorgensen K, Hanssen KF, Sandvik L (1988) The response of diabetic retinopathy to 41 months of multiple insulin injections, insulin pumps, and conventional insulin therapy. Arch Ophthalmol 106: 1241-1246

5. Diabetes Control and Complications Trial Research Group (1995) The implementation of treatment protocols in the Diabetes Control and Complications Trial. Diabetes Care 18: 361-376

6. Zinman B (1989) The physiologic replacement of insulin: an elusive goal. N Engl J Med 321: 363-370

7. UKPDS Study Group (1991) UK Prospective Diabetes Study (UKPDS) VIII Study, design, progress, and performance. Diabetologia 34: 877-890

8. The DCCT Research Group (1996) Lifetime benefits and costs of intensive therapy as practiced in the diabetes control and complications trial. JAMA 276: 1409-1415 